\title{
A NEW STRUCTURE FOR FEEDFORWARD ACTIVE NOISE CONTROL SYSTEMS WITH ONLINE SECONDARY-PATH MODELING
}

\author{
Muhammad Tahir Akhtar, Masahide Abe, and Masayuki Kawamata \\ Department of Electronic Engineering, \\ Tohoku University, Sendai 980-8579, JAPAN \\ Phone: +81-22-217-7095, Fax: +81-22-263-9169 \\ Email:akhtar@mk.ecei.tohoku.ac.jp
}

\begin{abstract}
This paper proposes a new structure for the feedforward active noise control (ANC) systems with online secondary path modeling. The proposed structure 1) uses the same error signal for updating the noise-control process as used for the modeling process, and 2) incorporates an averaging based filtered-reference algorithm in the noise-control process. The theoretical considerations are confirmed by the computer simulations, which show that the proposed structure achieves better performance than the existing methods. This improved performance is achieved at the expense of a slightly increased computational complexity.
\end{abstract}

\section{INTRODUCTION}

The most popular adaptation algorithm used for active noise control (ANC) applications is the FxLMS algorithm which is a modified version of the LMS algorithm [1]. The FxLMS algorithm is fairly robust to the modeling errors between the secondary path and the modeling filter; however, in general, the noise reduction performance is inferior to that under ideal conditions. Accordingly, we should use online identification of the secondary path characteristics to ensure the stability and to maintain the noise reduction performance when the secondary path is time varying [2].

The basic additive random noise technique for online secondary-path modeling in ANC systems is proposed by Eriksson et el. [3]. As shown in Fig. 1, this ANC system comprises two processes; a noise-control process (hereafter called the control process), and a secondary-path modeling process (hereafter called the modeling process). Improvements in this method have been proposed in Refs. [4] - [6]. These improved methods introduce another adaptive filter into the ANC system of Fig. 1, and hence the design complexity is increased.

In this paper we analyze the operation of the Eriksson's method and suggest modifications by observing various signals appearing in the system. This study results in an ANC

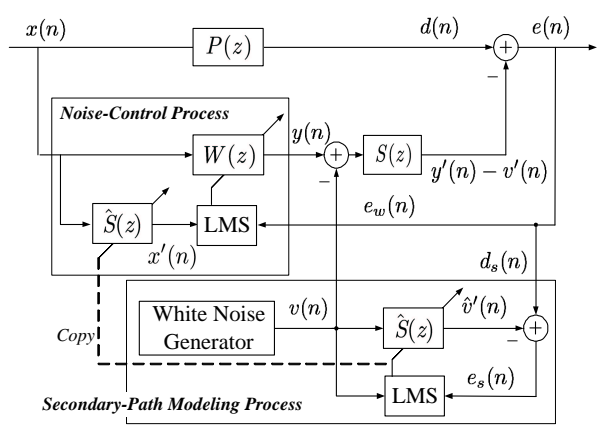

Fig. 1. Block diagram of FxLMS based ANC system with online secondary-path modeling (Eriksson's method).

system with online secondary path modeling that can achieve better performance than the existing improved methods.

The organization of this paper is as follows. Section 2 presents an overview of the existing methods for ANC systems with online secondary-path modeling. Section 3 explains the proposed method in connection to the operation of the Eriksson's method. Section 4 discusses the computational complexity issue and Section 5 details the simulation results.

\section{EXISTING METHODS}

Consider Eriksson's method for ANC systems with online secondary-path modeling, shown in Fig. 1. Assuming that the control filter $W(z)$ is an FIR filter of order $L$, the secondary signal $y(n)$ is expressed as

$$
y(n)=\boldsymbol{w}^{T}(n) \boldsymbol{x}_{L}(n)
$$

where $\boldsymbol{w}(n)=\left[w_{0}(n) w_{1}(n) \cdots w_{L-1}(n)\right]^{T}$ is the tap weight vector and $\boldsymbol{x}_{L}(n)=[x(n) x(n-1) \cdots x(n-L+1)]^{T}$ is the reference signal picked by the reference microphone. An internally generated zero mean white Gaussian noise signal, $v(n)$, uncorrelated with the reference noise $x(n)$, is injected at the output $y(n)$ of the control filter. The residual 


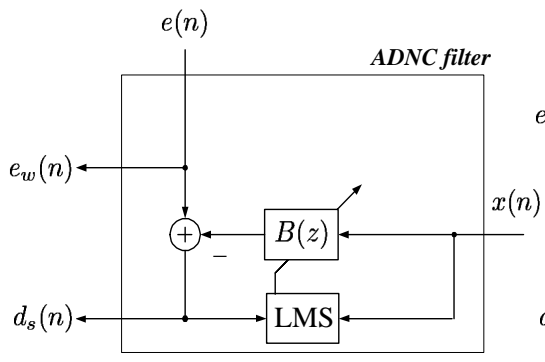

(a)

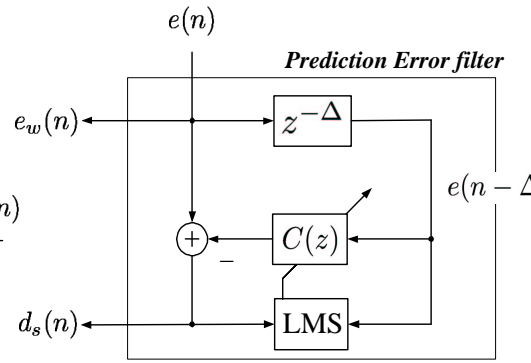

(b)

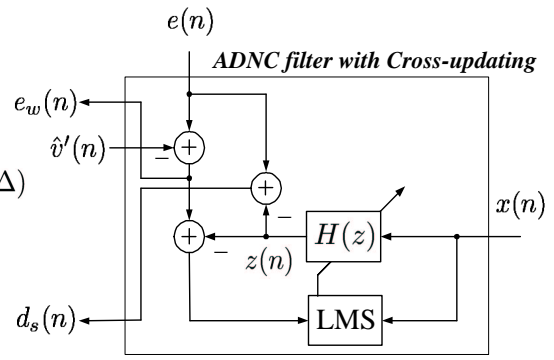

(c)

Fig. 2. Improved methods for online secondary-path modeling in ANC system of Fig. 1: (a) Bao's method, (b) Kuo's method, (c) Zhang's method

noise signal $e(n)$ is given as

$$
e(n)=d(n)-y^{\prime}(n)+v^{\prime}(n)
$$

where $d(n)=p(n) * x(n)$ is the primary disturbance signal at the error microphone, $y^{\prime}(n)=s(n) * y(n)$ is the secondary canceling signal, $v^{\prime}(n)=s(n) * v(n)$, * denotes the convolution operation, and $p(n)$ and $s(n)$ are the impulse responses of the primary path $P(z)$ and secondary path $S(z)$, respectively. The residual noise signal $e(n)$ is used as an error signal for the control process, and as a desired response for the modeling process, i.e., $e_{w}(n)=$ $d_{s}(n)=e(n)$. The coefficients of the control filter $W(z)$ are updated by the FxLMS algorithm:

$$
\begin{aligned}
\boldsymbol{w} & (n+1)=\boldsymbol{w}(n)+\mu_{w} e_{w}(n) \boldsymbol{x}^{\prime}(n) \\
& =\boldsymbol{w}(n)+\mu_{w} \boldsymbol{x}^{\prime}(n)\left[d(n)-y^{\prime}(n)+v^{\prime}(n)\right] \\
& =\boldsymbol{w}(n)+\mu_{w} \boldsymbol{x}^{\prime}(n) u(n)+\mu_{w} \boldsymbol{x}^{\prime}(n) v^{\prime}(n)
\end{aligned}
$$

where $\mu_{w}$ is the step size for the control process, $\boldsymbol{x}^{\prime}(n)$ is the reference signal $\boldsymbol{x}(n)$ filtered through the modeling filter $\hat{S}(z)$, and $u(n)=d(n)-y^{\prime}(n)$ is a component of the error signal due to cancelling noise only. We see that the control process is perturbed by an undesired term $\mu_{w} \boldsymbol{x}^{\prime}(n) v^{\prime}(n)$.

Assuming that $\hat{S}(z)$ is represented by an FIR filter of order $M$, the filtered-reference signal $x^{\prime}(n)$ is obtained as

$$
x^{\prime}(n)=\hat{\boldsymbol{s}}^{T}(n) \boldsymbol{x}_{M}(n)
$$

where $\hat{\boldsymbol{s}}(n)=\left[\hat{\boldsymbol{s}}_{1}(n) \hat{\boldsymbol{s}}_{2}(n) \cdots \hat{\boldsymbol{s}}_{M-1}(n)\right]^{T}$ is the impulse response of the modeling filter $\hat{S}(z)$ and $\boldsymbol{x}_{M}(n)=[x(n) x(n-$ 1) $\cdots x(n-M+1)]^{T}$. The LMS update equation for $\hat{S}(z)$ is given as

$$
\begin{aligned}
& \hat{\boldsymbol{s}}(n+1)=\hat{\boldsymbol{s}}(n)+\mu_{s} e_{s}(n) \boldsymbol{v}(n) \\
& \quad=\hat{\boldsymbol{s}}(n)+\mu_{s} \boldsymbol{v}(n)\left[d(n)-y^{\prime}(n)+v^{\prime}(n)-\hat{v}^{\prime}(n)\right] \\
& \quad=\hat{\boldsymbol{s}}(n)+\mu_{s} \boldsymbol{v}(n)\left[v^{\prime}(n)-\hat{v}^{\prime}(n)\right]+\mu_{s} \boldsymbol{v}(n) u(n)
\end{aligned}
$$

where $\mu_{s}$ is the step size of the modeling process, $\hat{v}^{\prime}(n)=$ $\hat{s}(n) * v(n)$ is an estimate of $v^{\prime}(n)$ obtained from the modeling filter, and $\boldsymbol{v}(n)=[v(n) v(n-1) \cdots v(n-M+1)]^{T}$.
Equation (5) shows that the performance of the modeling process is degraded by an undesired term $\mu_{s} v(n) u(n)$ and in the worst case the modeling process may diverge.

Bao's method is an attempt to improve the convergence of $\hat{S}(z)$ in the presence of $u(n)$ by the introduction of ADNC filter [4] in the modeling process. As shown in Fig. 2(a), the ADNC filter $B(z)$, excited by $x(n)$, cancels the interference introduced by $u(n)$ in the estimation of $S(z)$. The method proposed by Kuo et el. is shown in Fig. 2(b). Here the adaptive prediction-error filter $C(z)$, excited by delayed version of $e(n)$, predicts and hence cancels the interference caused by $u(n)$ [5]. This idea limits the use of Kuo's method to only narrowband ANC systems where the reference noise $x(n)$ is predictable.

These two approaches have greatly improved the convergence rate of the modeling process as compared with the Eriksson's method. It is important to note that in these methods the error signal used for the control filter is same as the residual error signal of the system, i.e., $e_{w}(n)=e(n)$. Hence no effort is made to improve the performance of the control process.

The problem of mutual interference between the control process and the modeling process (due to $e_{w}(n)=d_{s}(n)=$ $e(n))$ is considered in the Zhang's method (Fig. 2(c)) [6]. Here the control filter, modeling filter and ADNC filter are cross-updated to reduce the mutual coupling between the control process and modeling process [6]. As shown in Fig. 2(c), $e_{w}(n)$ and $d_{s}(n)$ are computed as

$$
\begin{aligned}
& e_{w}(n)=e(n)-\hat{v}^{\prime}(n)=u(n)+\left[v^{\prime}(n)-\hat{v}^{\prime}(n)\right](6) \\
& d_{s}(n)=e(n)-z(n)
\end{aligned}
$$

Here $e_{w}(n)$ is used as a desired signal for ADNC filter. When $\hat{S}(z)$ converges then $v^{\prime}(n) \approx \hat{v}^{\prime}(n)$ and $e_{w}(n) \approx$ $u(n)=d(n)-y^{\prime}(n)$. When $H(z)$ converges then $z(n) \approx$ $u(n)$ and hence $d_{s}(n) \approx v^{\prime}(n)$. Thus when $H(z)$ and $\hat{S}(z)$ converge, appropriate signals are generated and an improved performance is expected. The simulations conducted by Zhang et el. show that this structure gives the best performance of all previous designs [6]. 


\section{PROPOSED METHOD}

From the above discussion we see that all improved methods concentrate on the modeling process and control process is same as in the Eriksson's method. In the present study we consider improving the performance of the control process. The basic idea is that if the control process is efficient in its noise-reduction performance, then modeling process will in turn converge fast.

As shown in Fig. 1, the error signals for the control process and the modeling process are given as

$$
\begin{aligned}
& e_{w}(n)=e(n)=u(n)+v^{\prime}(n) \\
& e_{s}(n)=e(n)-\hat{v}^{\prime}(n)=u(n)+\left[v^{\prime}(n)-\hat{v}^{\prime}(n)\right](9)
\end{aligned}
$$

From these expressions we observe that:

- Both $e_{w}(n)$ and $e_{s}(n)$ contain $u(n)$, the error signal required for the noise control process. In $e_{w}(n), u(n)$ is corrupted by the component $v^{\prime}(n)$ and in $e_{s}(n)$ it is corrupted by the term $\left[v^{\prime}(n)-\hat{v}^{\prime}(n)\right]$.

- As compared with $e(n), e_{s}(n)$ is a better error signal for the control process, because $\left|v^{\prime}(n)-\hat{v}^{\prime}(n)\right|<$ $\left|v^{\prime}(n)\right|$ and when $\hat{S}(z)$ converges then (ideally) $v^{\prime}(n) \approx$ $\hat{v}^{\prime}(n) \Rightarrow\left[v^{\prime}(n)-\hat{v}^{\prime}(n)\right] \rightarrow 0$.

- Since $v(n)$ is a white Gaussian noise of zero mean hence both $v^{\prime}(n)$ and $\left[v^{\prime}(n)-\hat{v}^{\prime}(n)\right]$ are random in nature and can be averaged out.

On the basis of the above analysis two modifications are suggested to the Eriksson's method. First, using $e_{s}(n)$ as an error signal for both the control process and the modeling process, i.e., $e_{w}(n)=e_{s}(n)=e(n)-\hat{v}^{\prime}(n)$. Second, replacing FxLMS algorithm with the adaptive filtering with averaging (AFA) [7] based filtered-x (FxAFA) algorithm. The proposed ANC system is shown in Fig. 3.

The FXAFA algorithm [8] is a modified version of the FxLMS algorithm, where averaging is incorporated with both the iteration vector and the observation vector. Due to averaging the random fluctuations in the learning curves are reduced and hence iterations move towards the optimal solution at a faster rate. Referring to the FxLMS algorithm in Eq. (3), the FxAFA algorithm can be formulated as given in Eq. (10).

$\boldsymbol{w}(n+1)=\overline{\boldsymbol{w}(n)}+\frac{1}{n^{\gamma}} \sum_{k=1}^{n} \mu_{w} e_{w}(k) \boldsymbol{x}^{\prime}(k) ; \quad 1 / 2<\gamma<1$

where $\overline{\boldsymbol{w}(n)}=\frac{1}{n} \sum_{k=1}^{n} \boldsymbol{w}(k)$. It is important to note that computing the running average of the data does not put so much computational burden since averages can be calculated recursively [8]. Now better noise reduction performance is expected and ANC will reduce the residual noise component $u(n)$ at fast convergence rate. This means that the modeling process is expected to converge fast now.
Table 1. Computational complexity (Number of Multiplications/Iterations) comparison of the proposed method with the existing methods.

\begin{tabular}{|l|l|c|c|}
\hline \hline & $\begin{array}{l}\text { Analytical } \\
\text { Expression }\end{array}$ & $\begin{array}{c}L=M=64, \\
N=32\end{array}$ & $\begin{array}{c}L=M=128, \\
N=64\end{array}$ \\
\hline Eriksson's Method & $2 L+3 M+2$ & 322 & 642 \\
Improved Methods & $2 L+3 M+2 N+3$ & 387 & 899 \\
Proposed Method & $6 L+3 M+2$ & 578 & 1154 \\
\hline
\end{tabular}

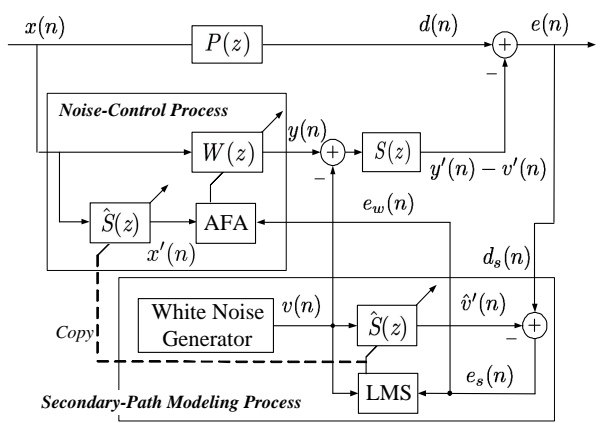

Fig. 3. Proposed method for ANC systems with online secondarypath modeling.

\section{COMPUTATIONAL COMPLEXITY}

Table 1 presents the computational complexity (multiplications per iteration) comparison of the proposed methods with the existing methods. It is assumed that three adaptive filters, $B(z), C(z)$ and $H(z)$ in Bao's method, Kuo's method and Zhang's method, respectively, are selected of order $N$. Hence these methods (called Improved Methods in Table 1) have the same computational complexity.

In improved methods, the order of the third filter, $N$, is usually selected less than $L$ (a thumb rule is $N \leq L / 2$ ). If a large value for $N$ is selected then the convergence of the third filter is slow, and overall performance of the ANC system is degraded. With this constraint, the analytical expressions in Table 1 show that the computational complexity of the proposed method is greater than the existing schemes. To get a clear picture, a few numerical examples are also presented in Table 1.

\section{SIMULATIONS}

In this section we compare the performance of the proposed method with the Eriksson's method and Zhang's method. The performance comparison is done on the basis of the relative modeling error $(R M E)$ being defined as $R M E=$ $10 \log _{10}\left[\Sigma_{i=0}^{M-1}\left\{s_{i}(n)-\hat{s}_{i}(n)\right\}^{2} / \Sigma_{i=0}^{M-1} s_{i}^{2}(n)\right]$. For the primary acoustical path $P(z)$ and the secondary path $S(z)$ the experimental data provided by [1] is used, where both are 
modeled by IIR filters of order 25 . We truncate the impulse responses of the acoustic paths to get FIR (order 64) representation. The modeling filter $\hat{S}(z)$ and control filter $W(z)$, respectively, are FIR filters of order 64. The ADNC filter $H(z)$ in Zhang's method is selected as an FIR filter of order 32. All initial values $\boldsymbol{w}(0), \hat{\boldsymbol{s}}(0)$, and $\boldsymbol{h}(0)$ are set to zero. The sampling frequency of $4 \mathrm{kHz}$ is used. All the results shown below are the average of 25 experiments.

\subsection{Case 1}

For a reference signal, the sinusoid of $330 \mathrm{~Hz}$ is added to the sinusoid of $300 \mathrm{~Hz}$, and then variance of the resulting signal is adjusted to two. To keep the low steady state residual noise, a zero-mean white Gaussian noise of variance 0.05 is used in the modeling process. The parameters are adjusted for fast and stable convergence and are summarized in Table 2. In Fig. 4(a), are shown the curves of the relative modeling error, $R M E$, for the proposed methods in comparison to the existing methods. Here Eriksson modified method is Eriksson's method with $e_{w}(n)=e_{s}(n)=e(n)-\hat{v}^{\prime}(n)$. This structure is obtained by replacing FxAFA algorithm with FxLMS algorithm in ANC system of Fig. 3. We see that the Eriksson modified method beats the Eriksson's method, and the proposed method gives the best result of all.

\subsection{Case 2}

In this case a sinusoid of $450 \mathrm{~Hz}$ is added to the sinusoid of $300 \mathrm{~Hz}$, and then variance of the resulting signal is adjusted to two. As in Case 1, a zero-mean white Gaussian noise of variance 0.05 is used in the modeling process. Fig. 4(b) shows the curves of the relative modeling error, $R M E$, for various methods. We see that proposed method gives the best performance.

\section{CONCLUDING REMARKS}

In this paper we have proposed a new structure for the feedforward ANC systems with improved online secondary-path modeling. Here an effort is made to improve the control process, so that better noise reduction is achieved and hence modeling process converges fast. The proposed structure uses two adaptive filters, $W(z)$ and $\hat{S}(z)$, to perform the noise-control and secondary-path modeling simultaneously. This is in contrast to existing improved methods which use three adaptive filters.

In the noise-control process, running averages of data are incorporated with the FxLMS algorithm. Due to this averaging the proposed FxAFA algorithm has long memory, and hence poor tracking properties. Improving the tracking properties of the proposed ANC system is a task of future work.
Table 2. Simulations parameters for computer experiments.

\begin{tabular}{|c|c|c|}
\hline \hline Case & $\begin{array}{c}\text { Eriksson's Method } \\
\left(\mu_{w}, \mu_{s}\right)\end{array}$ & $\begin{array}{c}\text { Eriksson Modified } \\
\text { Method }\left(\mu_{w}, \mu_{s}\right)\end{array}$ \\
\hline 1 & $1 \times 10^{-5}, 5 \times 10^{-4}$ & $2 \times 10^{-5}, 7 \times 10^{-4}$ \\
2 & $5 \times 10^{-5}, 5 \times 10^{-4}$ & $5 \times 10^{-5}, 7 \times 10^{-4}$ \\
\hline \hline & Zhang's Method & Proposed Method \\
& $\left(\mu_{w}, \mu_{s}, \mu_{h}\right)$ & $\left(\mu_{w}, \gamma, \mu_{s}\right)$ \\
\hline 1 & $2 \times 10^{-5}, 2 \times 10^{-3}, 2 \times 10^{-3}$ & $5 \times 10^{-5}, 0.5,2 \times 10^{-3}$ \\
2 & $2 \times 10^{-5}, 5 \times 10^{-3}, 2 \times 10^{-3}$ & $5 \times 10^{-4}, 0.5,5 \times 10^{-3}$ \\
\hline \hline
\end{tabular}

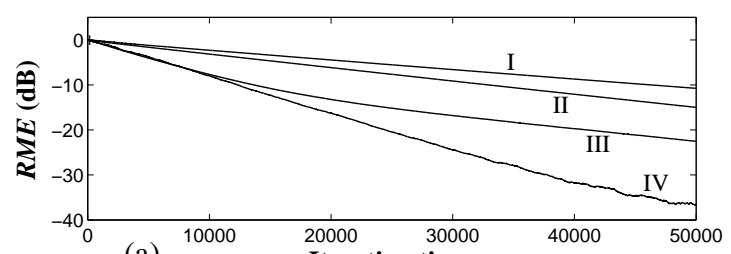

(a)

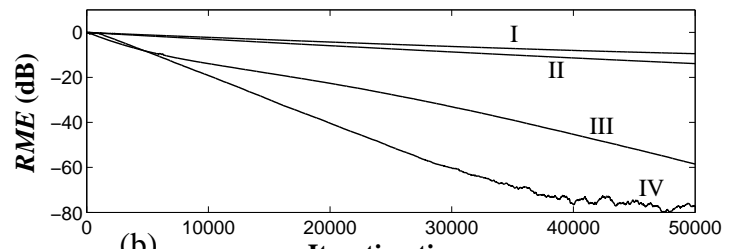

(b)

Fig. 4. Relative modeling error $R M E$ versus iteration time $n$; (a) Case 1, (b) Case 2: (I) Eriksson's method, (II) Eriksson modified method, (III) Zhang's method, (IV) Proposed method.

\section{REFERENCES}

[1] S.M. Kuo and D.R. Morgan, Active Noise Control Systems-Algorithms and DSP Implementation. New York: Wiley, 1996.

[2] N. Saito, and T. Sone, "Influence of modeling error on noise reduction performance of active noise control systems using filtered-x LMS algorithm,” J. Acoustic. Soc. Jpn. (E), vol. 17, no. 4, pp. 195-202, 1996.

[3] L. J. Eriksson, and M. C. Allie, "Use of random noise for on-line transducer modeling in an adaptive active attenuation system," J. Acoust. Soc. Am., vol. 85, issue 2, pp. 797-802, February 1989.

[4] C. Bao, P. Sas, and H. V. Brussel, "Adaptive active control of noise in 3-D reverberant enclosure," J. Sound Vibr., vol. 161, no. 3, pp. 501-514, 1993.

[5] S.M. Kuo, and D. Vijayan,"A secondary path modeling technique for active noise control systems," IEEE Trans. Speech Audio Processing, vol. 5, no. 4, pp. 374-377, July 1997.

[6] M. Zhang, H. Lan, and W. Ser, "Cross-updated active noise control system with online secondary path modeling," IEEE Trans. Speech, Audio Proc., vol. 9, no. 5, pp. 598-602, July 2001.

[7] G. Yin, "Adaptive filtering with averaging," in Adaptive Control, Filtering, and Signal Processing, K.J. Åström, G.C. Goodwin, and P.R. Kumar, Ed. New York: Springer-Verlog, 1995.

[8] A.M. Tahir, M. Abe, and M. Kawamata, "On use of averaging in FxLMS algorithm for single-channel feedforward ANC systems," Proceedings IEEE ISCAS 2003, vol. IV, pp. 389-392, May 2003. 